\title{
FIELD DEMONSTRATION OF LASER SCANNING FOR EXCAVATION MEASUREMENT ${ }^{\dagger}$
}

\author{
Geraldine S. Cheok ${ }^{\ddagger}$, Robert R. Lipman, Christoph Witzgall, \\ Javier Bernal, William C. Stone
}

\author{
National Institute of Standards and Technology \\ 100 Bureau Drive \\ Gaithersburg, MD 20899, USA
}

\begin{abstract}
The use of a scanning laser to measure terrain changes due to excavation at a construction site is described. The objective at this phase of the project is to develop the tools necessary to measure terrain changes in real-time. This paper focuses on adaptations required to extend previously developed scanning procedures and post-processing algorithms for an indoor laboratory environment to a large outdoor area such as a construction site. The challenges encountered, techniques that worked or didn't work, and lessons learned are discussed.
\end{abstract}

Keywords: Construction, excavation, LADAR, laser sensor, terrain mapping, volume calculations.

\section{INTRODUCTION}

This paper describes the work at the National Institute of Standards and Technology (NIST) in the Non-Intrusive Scanning Technologies for Construction Assessment Project. The initial proof-of-concept phase of this project is described in an earlier paper [1]. The objective of the initial phase was to show that a scanning laser, LADAR (laser distance and ranging), could be used to rapidly track terrain changes due to excavation at a construction site and that procedures and methods could be developed to display the results in real time. This was accomplished in a controlled indoor environment. This phase of the project involves extending the procedures and methods from an indoor environment to an outdoor, uncontrolled environment - a construction site.

The process of terrain tracking involves obtaining the data with a laser scanner, transferring data, post-processing of data, and displaying the data. This paper will focus on how the data was obtained and the post-processing of the data with the main emphasis on the latter subject. The discussion will include challenges encountered, lessons learned during this field demonstration, and areas in need of research.

\section{FIELD SCANNING}

\subsection{Background}

The research reported herein was conducted at a construction site located on the NIST campus in Gaithersburg, Maryland. The construction project, which began in December 1999, involves earthmoving and the assembly of an emissions control system for a fire testing facility. Figure 1 shows the terrain prior to construction. A Riegl scanner ${ }^{1}$, LPM98, was used to scan the terrain. Further details of this scanner may be found in [1].

To track the terrain changes, the scanned data (point clouds) were obtained from two locations around the construction site at the end of each work day which involved new excavation: on the roof of a building adjacent to the construction site and from a steel pole located within the construction site just beyond the construction boundary. The rooftop location was selected because it allowed for the least obstructed view and was out of the way of construction equipment. The field pole was set in concrete and the top of the pole was approximately $2.7 \mathrm{~m}$ above ground level. This height was chosen because an operator could use a ladder to install the scanner on the pole without the need for a mechanical lift, yet was high enough to provide better viewing of the site. Digital photos, from each location, were taken of the construction site when the scans were obtained. When the scan was completed, the data were stored in a laptop and were

\footnotetext{
${ }^{1 \uparrow}$ Official contribution of the National Institute of Standards and Technology; not subject to copyright in the United States.

* Author to whom correspondence should be addressed: cheok@nist.gov
}

Certain company products are mentioned in the text in order to adequately specify the equipment used. In no case does such an identification imply recommendation or endorsement by the National Institute of Standards and Technology, nor does it imply that the products are necessarily the best available for the purpose. 
later transferred via FTP (file transfer protocol) to a remote computer for post-processing.

Post-processing involved initial registration of the two scans based on the known positions of the scanner, fine tuning the registration visually, determining the regions of interest (needs to be done only once), cropping the scanned data set to the regions of interest, creating a surface model from the scanned data, and computing a volume. Volume changes are needed to track the construction progress, amount of materials delivered, and for determining payment of tasks completed.

Two methods were used to compute the volume changes of the terrain from one day to another. One method to compute the volume is by finding the volume bounded by two surfaces: an elevated triangulated surface (representing the terrain) and a planar surface at an elevation below the triangulated surface. This procedure was performed for each day that a scan was obtained and the volume change between any two days is obtained by subtracting one volume from the other. The second method is similar to the first method except that the two bounding surfaces for a given day are extended to be closed triangulated surfaces in 3-D space. The tetrahedralized intersection of the two polyhedral regions enclosed by the two surfaces is then determined. The volume changes can be obtained by subtracting the intersection from the polyhedral regions enclosed by the first and second surfaces which yields the cut and fill volumes, respectively. Further details of these two methods may be found in [2].

After the scanned data has been postprocessed, surface models representing the terrain and quantities of dirt moved may be displayed on a web site. This allows for wide access of the information by interested parties such as contractors, engineers, and owners for remote tracking of the work progress.

\subsection{Post-Processing Data}

Prior to the commencement of any construction work, the terrain was scanned from 5 positions around the site to obtain an initial reference surface. The locations of these points were determined from a traditional total station survey. The surface was created from the combined point cloud data from seven scans (at two positions, two scans were obtained from each position). A truck was parked in the scanned area to aid in the registration of the seven scans as shown in Fig. 2. Care was taken to ensure that the truck would not be in an area where volume calculations would subsequently be important. This was because the points defining the truck would not be distinguishable from the points defining the terrain. Therefore, the surface generated from the combined point cloud would include the truck data and yield a "false" differential volume.
This presented the first challenge to scanning a construction site: the ability to identify points within a point cloud that define an object that is not part of the terrain and needs to be removed. A fully automated solution is a complex process and human assisted identification of the object is currently necessary. For the current work, a computer program was developed at NIST to eliminate points within a user defined rectangular area. The limits (starting and ending points) of this area are determined by the user after identifying the object in a 2-D triangulated mesh of the terrain. A more interactive solution would be to graphically view the point cloud, and remove the points defining the object from the data set.

Figure 3 shows the surface model of the initial terrain. As seen in Fig. 3, the buildings, trees and truck are not easily recognizable as such and prior knowledge or other visual aids are needed to identify them.

A second challenge was data registration. Initial transformations were performed using the known position of the scanner locations with further adjustments made using an interactive graphics program. This visual alignment of the scans works well for adjusting the $\mathrm{X}$ - and $\mathrm{Y}$-translations and rotations about the $\mathrm{Z}$-axis. Adjustments about the remaining degrees-of-freedom were not possible due to the inability to visually differentiate the points in the foreground and the background. To adjust the rotations about the $\mathrm{X}$ - and $\mathrm{Y}$-axes and the Ztranslations, 3-D surface models of the scans were used to aid in visual detection of any misalignments which show up as ridges along the scan path in the model. Most of the rotational adjustments ranged from about $0.1^{\circ}$ to $0.2^{\circ}$. Making these manual adjustments for each data set was very time consuming.

Based on this experience, the scanning techniques will require two types of registration. The first type of registration, spatial registration, aims at properly combining several scans taken from different vantage points of the same scene. The need for several vantage points is necessitated by inadvertent occlusions of the scene (e.g., machinery, components, and foliage). The second type of registration, temporal registration, is required if two surfaces representing the scene at different times are to be compared. Such a comparison is required if a timeline depicting scene changes is needed. Systematic targeting procedures with suitable targets (e.g., pyramid) is expected to be a viable solution for the registration of scans from different times.

A first attempt was made to develop an algorithm for automatic registration of a point cloud to a surface by minimizing the deviations between the point cloud and the surface. The program assumes that the scans were already initially transformed to a global coordinate system, i.e., close to "truth". This attempt was hampered by data noise. This method also assumes that one scan represents ground truth which is incorrect. The 
inclusion of targets with known geometry and position in the scene may aid in data registration and will be investigated in future efforts in this project.

The calculated volumes for selected dates are given in Table 1, and the terrain and surface models are shown in Fig. 4. For example, the second row represents the volume change between Mar. 7, 2000 and Mar. 6, 2000 and these are fill volumes (positive values). No uncertainty values can be associated with these values for two reasons. First, it was not possible to measure the actual volumes of the material added or removed. Second, there do not exist any accepted or standard test protocols for instrument calibration. In addition to procedures for traditional instrument calibration (range and pointing accuracy), it is important to recognize that calibration also includes the ability to determine measures of performance for the algorithms used to generate the 3-D surface models that are subsequently used in volumetric calculations. Registration errors will also have to be included when determining the combined uncertainty for the volume calculations. For these reasons, it is not possible to determine which of the two values obtained for the A2 region for Mar. $9 /$ Mar. 7 (Table 1) is more accurate. NIST has current programs working towards resolution of these calibration issues for LADAR-based terrain metrology.

\subsection{Problems Encountered in Field Scanning}

Several challenges were encountered when obtaining field scans. One was to supply power to the scanner when it was on top of the roof and in the field where AC power was not readily available. The scanner required a minimum of $11 \mathrm{~V} \mathrm{DC}$ to a maximum of $18 \mathrm{~V} \mathrm{DC}$ at $8 \mathrm{~A}$. The obvious solution was to use a battery for the power supply and an auto battery would meet these power requirements. A difficulty with the use of a battery is that it is heavy and very cumbersome to carry up and down from buildings and around the construction site. The batteries could not be left in place because they had to be recharged after each use. In addition to the battery, the scanner, laptop, and accessories had to be carried up to and down from the roof and to the field pole. A solution is to have multiple scanners and to leave the equipment in place inside weatherproof, auto-deploying poles which pop up several times daily, capture and transmit their data, then go into "sleep" mode and close their environmental enclosures. The advantages are elimination of daily set-up, alignment and leveling of the scanner. The disadvantages include the cost of purchasing multiple scanners (a factor that could be moderated by the development of low cost LADARs), the need to develop waterproof casings for the equipment, and the ability to secure the equipment from theft. In addition, storage temperatures of the instruments will have to be considered if the instrument were to be left in the field.

As expected, the scanned data taken from the roof had fewer obstructed regions than the similar data obtained from the pole in the field. Besides scanning from a much lower height, the situation in the field was further aggravated when a large pile of dirt was placed in front of the pole which further obstructed the scene. Also, access to the pole in the field was at times blocked by construction materials and machinery and the scanning equipment and accessories had to be handcarried to the pole.

As it was a relatively small construction project, we were able to work with the contractor to have most of the machinery parked beyond the areas of interest at the end of the day. This allowed the scanned areas to have fewer occluded regions and to be relatively free of obstructions. However, this approach may not be possible in larger construction projects. Here, the capacity for object identification would be required when post-processing the scanned data in order to remove any objects that are not part of the terrain.

Also, once the erection of the structure commences, further volume calculations may be problematic. As seen in Fig. 5, the surface generated from the scanned data, which include the structural steel, would adversely impact volume calculations. The problem is related to the problem of interference by movable objects. It points to the need for identification of surface "spikes" caused by small items such as scaffolding, poles, fencing, cables, and others.

As part of the initial instrument set-up, the scanner had to be set to point to a fixed start position. The corner of a building was used as the reference for sighting and fixing the horizontal position of the scanner. As mentioned earlier, scans were obtained after the workers left. However, when workers left late during the winter months, it was dark when the scan was obtained. This made it difficult to sight to the corner of the reference building and the light from truck headlights had to be used to illuminate the building. Insufficient light may not be a problem as less construction work occurs during the winter months and the scanner could be set-up earlier in the day when there is still light.

\section{DISCUSSION}

Based on this field demonstration, three areas in need of further research are identified: data registration, sensor calibration, and object identification. Two types of data registration are required: 1) spatial registration: aimed at combining several scans taken from different vantage points of the same scene and 2) temporal registration: when two surfaces representing the scene at different times are to be compared. In both 
cases, testing protocols are needed to assess the uncertainties caused by the registration process.

Similarly, two types of sensor calibration are required. The first type involves sensor evaluation. Establishing the accuracy of a sensor's range-measurement is straightforward. However, the calibration is more complex for scanning sensors as some scanners use lasers outside of the visible light range. Determination of the rotational accuracy of the sensor is, therefore, less straightforward.

Further, the accuracy of any numerical results based on the sensor data, needs to be ascertained. The accuracy of volume calculations are of particular interest. Therefore, the second type of calibration involves measures of performance for algorithms that are used to generate surfaces from the point clouds. These metrics are harder to establish. In general, performance is based on comparison with a reference model or ground truth. The accuracy of surface generation algorithms can be determined using objects of known shape and volume. However, can this "accuracy" for welldefined objects be extrapolated to highly irregular objects such as terrains?

Finally, object identification will facilitate the removal of objects that are not part of the terrain. Additionally, object identification will enable the tracking of parts around a construction site. There are several methods available to extract an object from a point cloud. The methods that are currently used to extract, replace, and/or remove objects within a scene are time consuming and require user intervention. Methods to extract objects automatically are, at this time, mainly research tools and have been successful for single objects in a scene. Other possible aids in object identification include the use of color, intensity of the returned signal, and pattern recognition. These methods do not identify objects per se, but are used to aid in object identification. Additional intelligence will have to supplied/added to pick out and extract the data (points) from a point cloud for further processing and to correctly identify the object. In the first instance, user intervention is the most likely source of this intelligence. In the latter instance, the intelligence could be in the form of user intervention, image processing algorithms (if a camera is used), and/or a database containing objects such as trucks, trees, and buildings that would most likely be found in a particular scene. If a database is utilized to provide the intelligence, probability analyses will have to be performed to determine confidence limits.

\section{SUMMARY}

The experiences of using a LADAR to track terrain changes at a construction site are documented. Some of the difficulties encountered with field scanning include getting power to the scanner in the field, water proofing equipment, and finding a location that resulted in the least obstructed view.

When post-processing the data, registration of scanned data taken from different locations and at different times required intensive manual intervention. Also, data points that define objects were manually identified and removed. For laser scanning to be a viable technology, these tasks, data registration and object identification, will have to be automated to a greater degree in order to minimize user intervention. Metrics for sensor calibration will also have to be developed. These metrics are needed for the instrument calibration, range and rotational accuracy, as well as for the methods and algorithms used to generate the surface model.

\section{REFERENCES}

[1] Cheok, G. S., Stone, W. C., Lipman, R. R., and Witzgall, C., "LADARS for Construction Assessment and Update," to be published in Automation in Construction journal.

[2] Cheok, G. S., Lipman, R. R., Witzgall, C., Bernal, J., Stone, W. C., "NIST Construction Automation Program Report No. 4: Non-Intrusive Scanning Technology for Construction Status Determination," NISTIR 6457, National Institute of Standards and Technology, Gaithersburg, MD, January, 2000, 93 pp.

Table 1. Computed Volume Changes ${ }^{\sharp}$ for Selected Dates

\begin{tabular}{|c|c|c|c|c|}
\hline \multirow{2}{*}{$\begin{array}{c}\text { Selected Dates } \\
\text { Second date / First date }\end{array}$} & Triangulation & Tetrahedralization & Triangulation & Tetrahedralization \\
\cline { 2 - 5 } & +340.6 & +339.7 & +35.0 & +35.2 \\
\hline Dec. 17 / Initial Terrain & +287.1 & +293.3 & +111.5 & +111.7 \\
\hline Mar. 7 / Mar. 6 & -19.8 & -43.0 & -38.9 & -39.0 \\
\hline Mar. 9 / Mar. 7 & & $\mathrm{A}^{\S}$ Region $\left(\mathrm{m}^{3}\right)$ \\
\hline
\end{tabular}

$\S$ A2 region comprises of the area $(67 \mathrm{~m} \times 50 \mathrm{~m})$ depicted by the surface model shown in Fig. 4 . A3 region comprises a region $31 \mathrm{~m} \times 33 \mathrm{~m}$ and is shown in Fig.4.

Volume change $=$ Second date minus first date. A positive value indicates a fill volume and a negative value indicates a cut volume. 


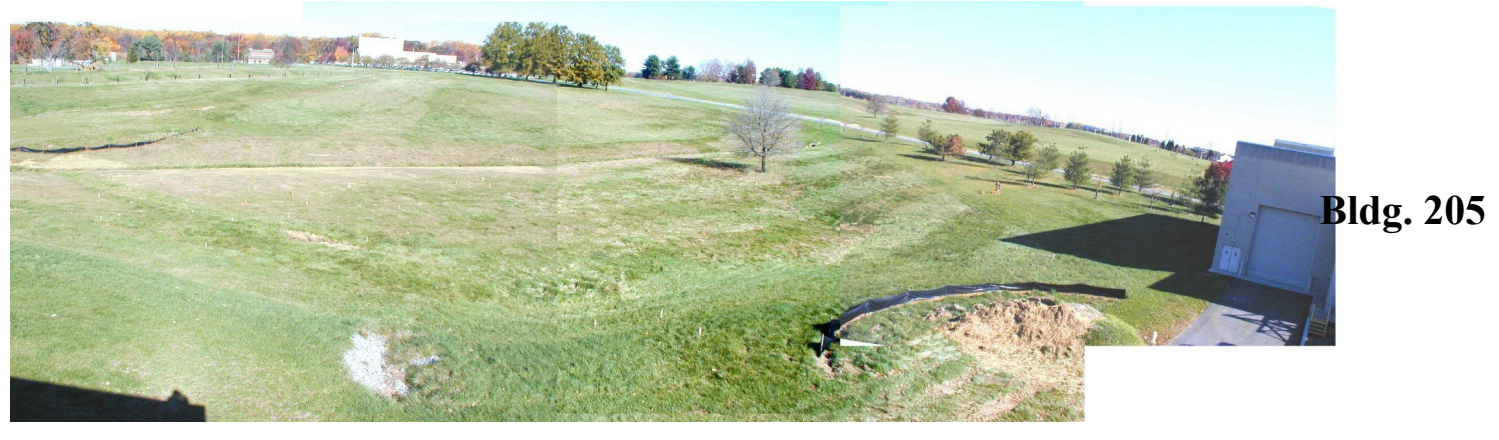

Figure 1. Initial Terrain of Construction Site.

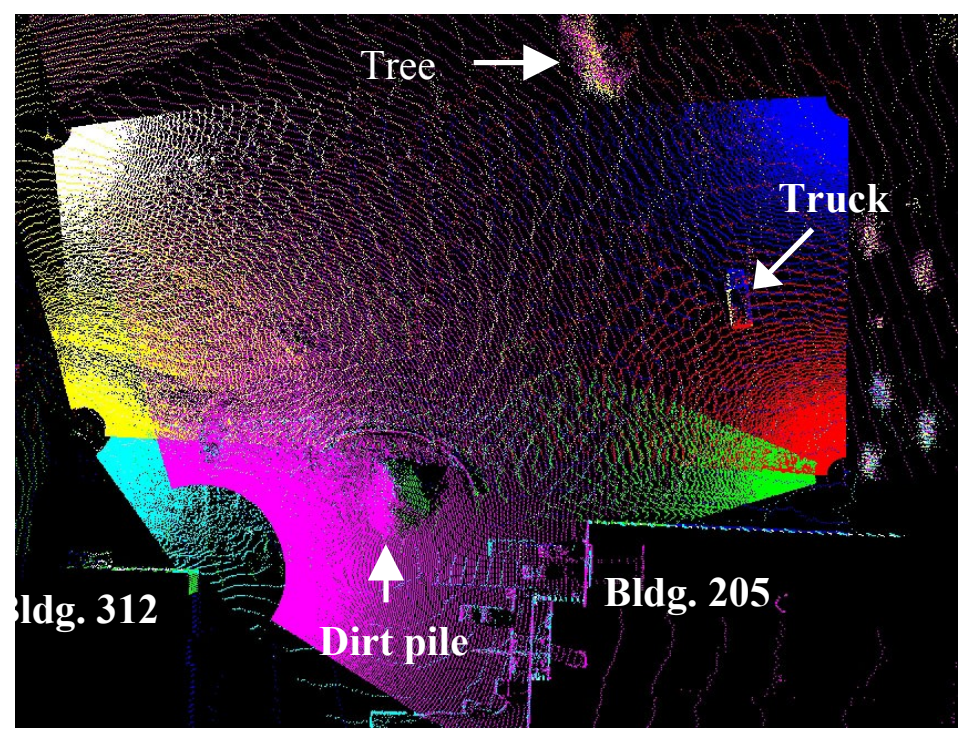

Figure 2. Point cloud of Initial Terrain (Truck driven into scene for registration purposes).

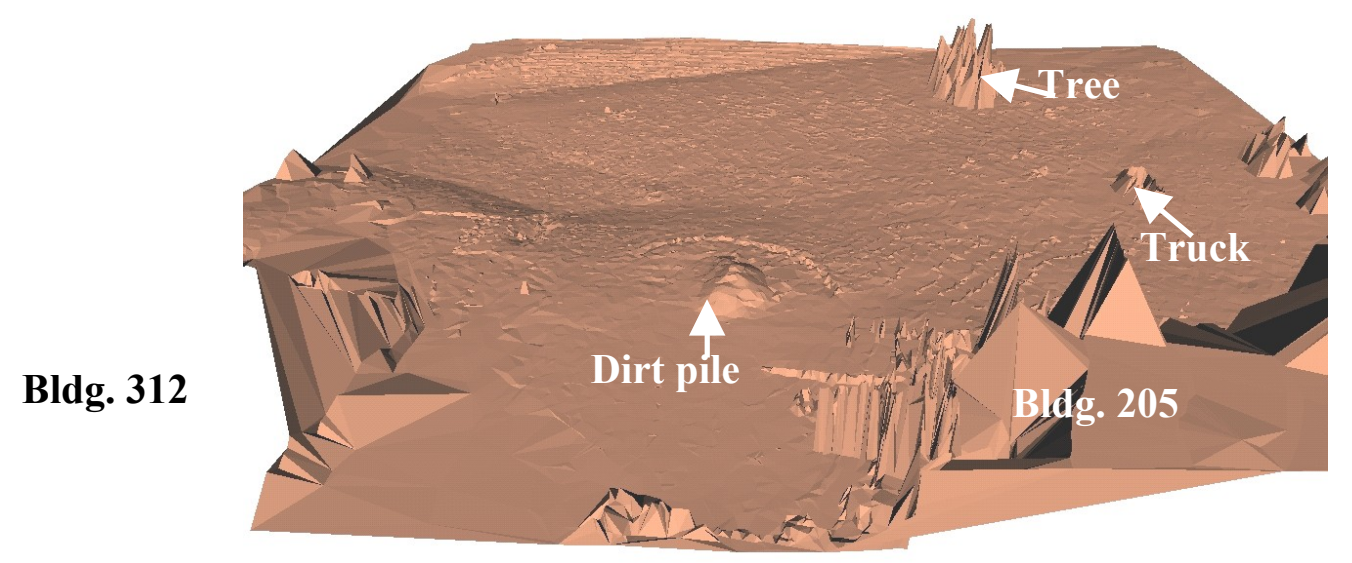

Figure 3. Surface Model of Initial Terrain. 


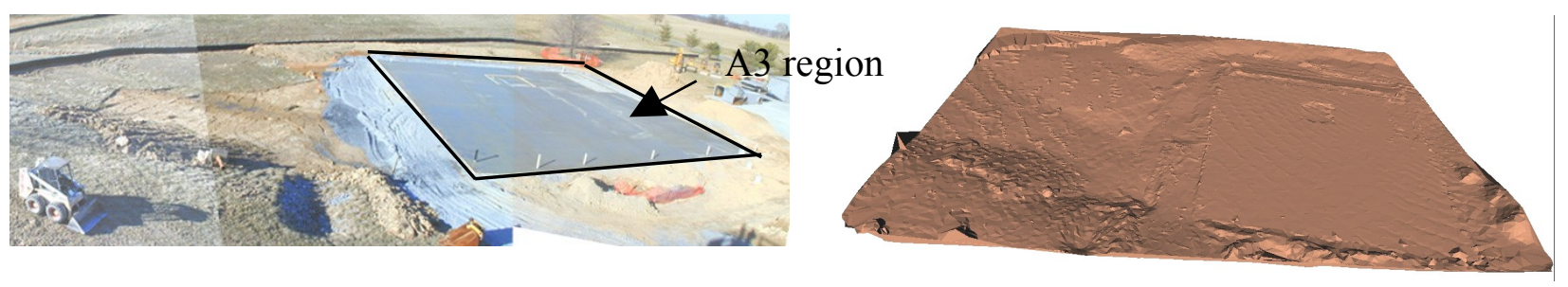

a. March 7, 2000
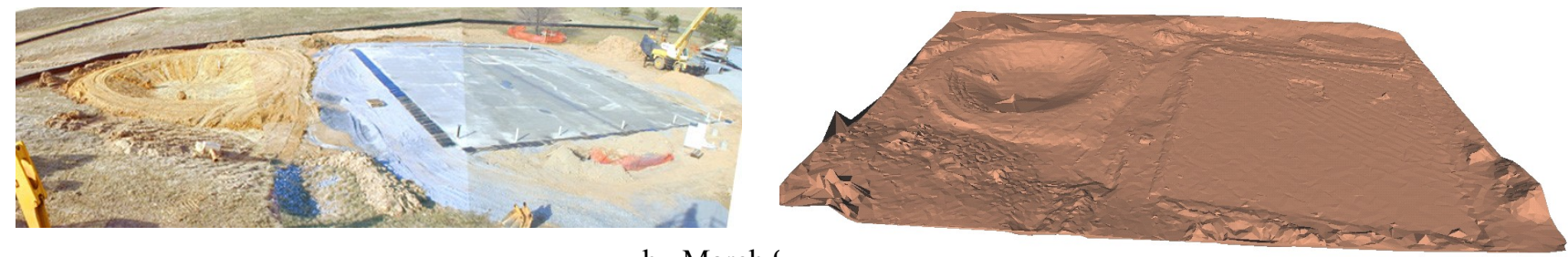

b. March Y, LuvU

Figure 4. Terrain and Surface Model (A2 region). Encompassed A2 region: $67 \mathrm{~m} \mathrm{x} 50 \mathrm{~m}$.
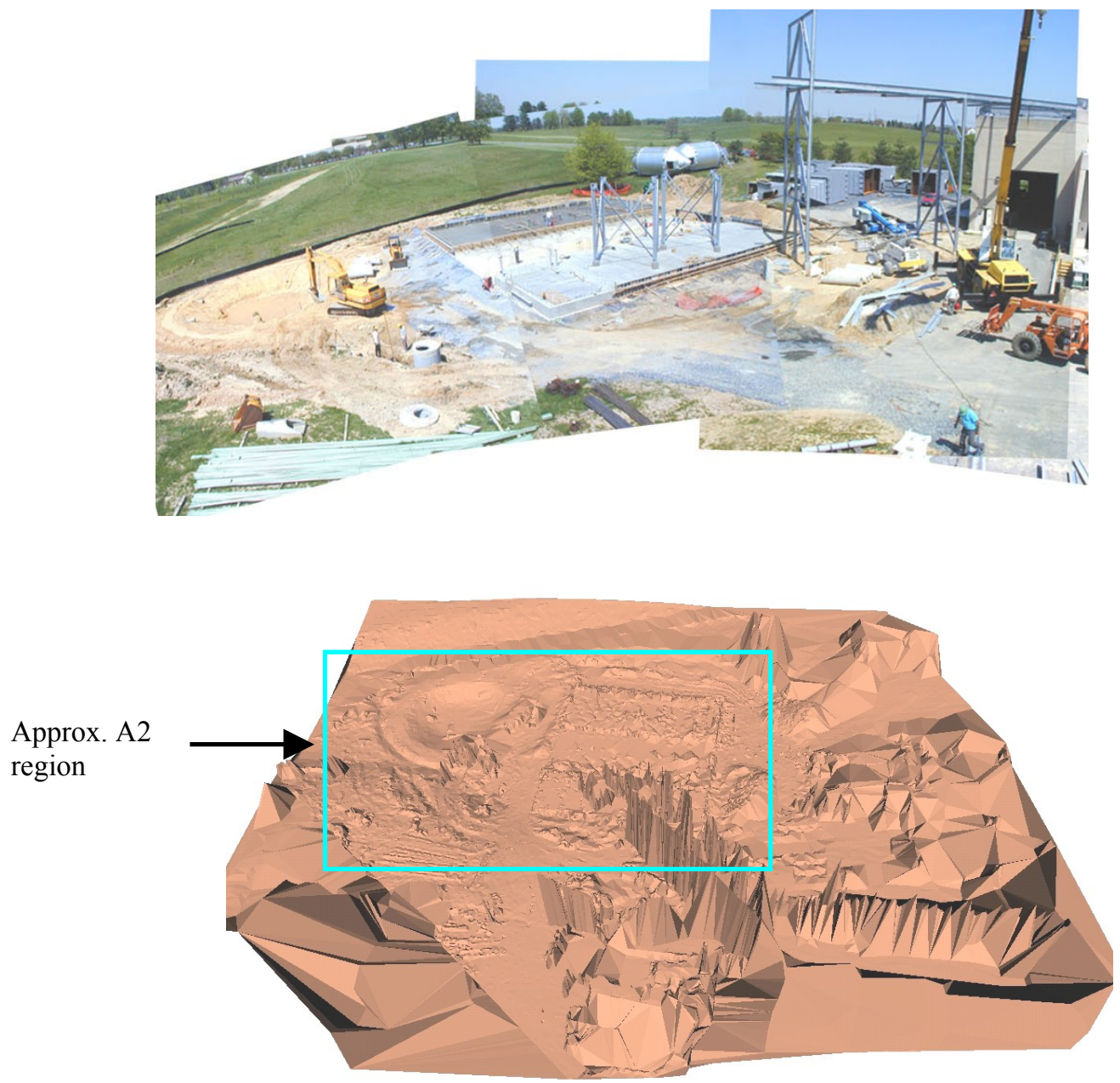

Figure 5. Surface "Spikes" Created by Structural Steel. Note that the area encompassed in this figure is larger than that shown in Fig. 4 and is $120 \mathrm{~m} \times 100 \mathrm{~m}$. 